\title{
Riemerella anatipestifer GIdM is required for bacterial gliding motility, protein secretion, and virulence
}

\author{
Zongchao Chen ${ }^{1+}$, Xiaolan Wang ${ }^{1 \dagger}$, Xiaomei Ren ${ }^{1}$, Wenlong Han ${ }^{1}$, Kanwar Kumar Malhi ${ }^{1}$, Chan Ding ${ }^{1}$ \\ and Shengqing $Y u^{1,2^{*}}$
}

\begin{abstract}
Riemerella anatipestifer is a major pathogenic agent of duck septicemic and exudative diseases. Genetic analyses suggest that this pathogen has a novel protein secretion system, known as the "type IX secretion system" (T9SS). We previously reported that deletion of the AS87_RS08465 gene significantly reduced the bacterial virulence of the R. anatipestifer strain Yb2, but the mechanism remained unclear. The AS87_RS08465 gene is predicted to encode the gliding motility protein GldM (GIdM) protein, a key component of the T9SS complex. In this study, Western blotting analysis demonstrated that $R$. anatipestifer GIdM was localized to the cytomembrane. Further study revealed that the adhesion and invasion capacities of the mutant strain RA2281 (designated Yb2 $\Delta g / d M$ ) in Vero cells and the bacterial loads in the blood of infected ducks were significantly reduced. RNA-Seq and PCR analyses showed that six genes were upregulated and five genes were downregulated in the mutant strain $\mathrm{Yb} 2 \triangle g l d M$ and that these genes were mainly involved in the secretion of proteins. Yb2 $\triangle$ gld $M$ was also found to be defective in gliding motility and protein secretion. Liquid chromatography-tandem mass spectrometry analysis revealed that nine of the proteins had a conserved T9SS C-terminal domain and were differentially secreted by Yb2 $\triangle$ gldM compared to Yb2. The complementation strain cYb2 $\triangle g / d M$ recovered the adhesion and invasion capacities in Vero cells and the bacterial loads in the blood of infected ducks as well as the bacterial gliding motility and most protein secretion in the mutant strain Yb2 $\triangle g / d M$ to the levels of the wild-type strain Yb2. Taken together, these results indicate that $R$. anatipestifer GldM is associated with T9SS and is important in bacterial virulence.
\end{abstract}

\section{Introduction}

Riemerella anatipestifer is a causative pathogen of diseases in ducks, geese, turkeys, and various other domestic and wild birds [1]. Infected ducks show clinical signs of lethargy, diarrhea, and respiratory and nervous symptoms, all of which cause serious economic losses in the duck industry [2]. Several virulence factors of $R$. anatipestifer have been identified, including CAMP cohemolysin, OmpA, glycosyl transferase, nicotinamidase PncA, VapD and other factors associated with lipopolysaccharide synthesis and iron acquisition [2-10]. However, the

\footnotetext{
*Correspondence: yus@shvri.ac.cn

†Zongchao Chen and Xiaolan Wang contributed equally to this work

${ }^{1}$ Shanghai Veterinary Research Institute, Chinese Academy of Agricultural

Sciences (CAAS), Shanghai, China

Full list of author information is available at the end of the article
}

mechanisms of $R$. anatipestifer virulence are not completely understood, which hinders the development of an efficient strategy to control this disease.

Riemerella anatipestifer is a member of the phylum Bacteroidetes. A novel protein secretion system, known as the "type IX secretion systems" (T9SSs) or "Por secretion system", has recently been frequently found in members of the phylum Bacteroidetes [11, 12]. Many virulence factors of pathogenic bacteria are either secreted proteins or the secretion systems themselves [13]. T9SS is associated with bacterial gliding motility and protein secretion and is considered to be a virulence factor in many pathogens $[14,15]$. Genetic analyses have shown that GldK, GldL, GldM, GldN, SprA, SprE, SprT, PorU, and PorV are components of T9SS in Flavobacterium johnsoni [16-19]. The proteins secreted

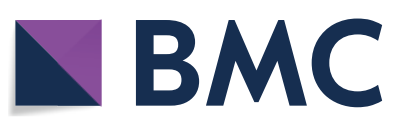

(c) The Author(s) 2019. This article is distributed under the terms of the Creative Commons Attribution 4.0 International License (http://creativecommons.org/licenses/by/4.0/), which permits unrestricted use, distribution, and reproduction in any medium, provided you give appropriate credit to the original author(s) and the source, provide a link to the Creative Commons license, and indicate if changes were made. The Creative Commons Public Domain Dedication waiver (http://creativecommons.org/ publicdomain/zero/1.0/) applies to the data made available in this article, unless otherwise stated. 
by T9SSs have a typical $\mathrm{N}$-terminal signal peptide and traverse the cytoplasmic membrane into the periplasm via the general secretion $(\mathrm{Sec})$ system. The proteins also typically have conserved C-terminal domains (CTDs) that target them to T9SS for secretion across the outer membrane [20]. SprT, encoded by the $\operatorname{spr} T$ gene, is a T9SS protein involved in protein secretion in $R$. anatipestifer. T9SS is functional in $R$. anatipestifer and contributes to its virulence by exporting key proteins [21]. The T9SS component GldM is required for bacterial gliding motility and the secretion of the cell-surface motility adhesins SprB and RemA in F. johnsoniae [22]. GldM is also required for the secretion of ChiA, which digests colloidal chitin and many other proteins $[17,18$, 23]. Sequence analyses have shown that $R$. anatipestifer GldM has a single predicted transmembrane helix near the $\mathrm{N}$-terminus that is highly conserved, suggesting that it functions beyond simple membrane anchoring, possibly in protein secretion and/or bacterial motility $[19,24]$.

Genetic techniques developed for $R$. anatipestifer have been used to identify many virulence genes [25]. We previously generated a virulence-attenuated mutant of strain $\mathrm{Yb} 2$, in which the $\mathrm{Tn} 4351$ transposon was inserted into the AS87_08795 gene. In this study, this mutant strain was shown to be defective in gliding motility and protein secretion. We also demonstrated that the AS87_08795 gene encodes a T9SS component that is involved in the virulence of $R$. anatipestifer.

\section{Materials and methods \\ Ethics statement}

The study protocol was approved by the Institutional Animal Care and Use Committee of Shanghai Veterinary Research Institute, the Chinese Academy of Agricultural Sciences (approval no. Shvri-po-2017090877), and was conducted in strict accordance with the recommendations outlined in the Guide for the Care and Use of Laboratory Animals. One-day-old Cherry Valley ducks were obtained from Zhuang Hang Duck Farm (Shanghai, China) and housed in cages at a controlled temperature of $28-30{ }^{\circ} \mathrm{C}$ under biosafety conditions, with water and food provided ad libitum.

\section{Bacterial strains, plasmids, and culture conditions}

The bacterial strains and plasmids used in this study are listed in Table 1. $R$. anatipestifer serotype 2 strain $\mathrm{Yb} 2$ is the wild-type strain. The $R$. anatipestifer strains were grown at $37{ }^{\circ} \mathrm{C}$ in tryptic soy broth medium (TSB, Difco, Franklin Lakes, NJ, USA). To prepare the solid tryptic soy agar (TSA) medium, $1.5 \mathrm{~g}$ of agar was added to $100 \mathrm{~mL}$ of TSB. The Escherichia coli-F. johnsoniae shuttle plasmid

Table 1 Strains, plasmids, and primers used in this study

\begin{tabular}{|c|c|}
\hline Strains, plasmids, primers & Characteristics \\
\hline Yb2 & Riemerella anatipestifer serotype 2 strain \\
\hline Escherichia colis17-1 & $\lambda$ pirhsdR pro thi; chromosomally integrated RP4-2, Tc::Mu Km::Tn7 \\
\hline $\mathrm{Yb} 2 \Delta g / d M$ & Tn4351 insertion mutant of $R$. anatipestifer Yb2, gldM::Tn \\
\hline $\mathrm{cYb} 2 \Delta g / d M$ & Mutant Yb2 $\triangle$ gldM carrying plasmid pCP29-gldM \\
\hline pCP29 & ColE1 ori (pCP1 ori), $A p^{r}\left(E m^{r}\right)$; E. coli-F. johnsoniae shuttle plasmid \\
\hline pCP29-gldM & pCP29 containing ompA promoter and gldM ORF, $c f x A\left(A p^{r}\right)$ \\
\hline \multicolumn{2}{|l|}{ Primers } \\
\hline AS87_RS08465-F & 5'-ATGGCAAAGGAAAAATT-3' \\
\hline AS87_RS08465-R & 5'-CTGAACATTTATCACTACTGGAG-3' \\
\hline ompA promoter $\mathrm{P} 1$ & 5'-CAGGTACCAGCTAAAATTTTGGCAGTAAC-3' (Kpnl site underlined) \\
\hline ompA promoter $\mathrm{P} 2$ & 5'-CGACTCGAGCATTCCAATTCTCTTATTATC-3' (Xhol site underlined) \\
\hline gldM-orf-F & 5'-CAGGTACCATGGCAAAGGAAAAATT-3' (Kpnl site underlined) \\
\hline gldM-orf-R & 5'-CGACTCGAGCTGAACATTTATCACTACTGGA-3' (Xhol site underlined) \\
\hline gldM-pro-F & 5'-CGGGATCCATTATACGCTCTTACAATGATAC-3' (BamHI site underlined) \\
\hline gldM-pro-R & 5'-GCGTCGACCTGAACATTTATCACTACTGGAG-3' (Sall site underlined) \\
\hline RA Idh-F & 5'-ATGAATTATTTTAAACTGCT-3' \\
\hline RA Idh-R & 5'-TTAGTCTAATTTCTGTATAT-3' \\
\hline RA 165 rRNA-F & 5'-TCTAAAATGAGATGTTCCA-3' \\
\hline RA 16 S rRNA-R & 5'-ACGAAAGCGTGGGGAGTGG-3' \\
\hline $\operatorname{Tn} 4351-\mathrm{F}$ & 5'-TGGCACCTTTGTGGTTCTTAC-3' \\
\hline Tn4351-R & 5'-GAGAGACAATGTCCCCCTTTC-3' \\
\hline
\end{tabular}

ORF: open reading frame, $c f x A$ : cefoxitin-resistance gene. 
pCP29 and E. coli strain S17-1 were kindly provided by Mark J. McBride (University of Wisconsin-Milwaukee, Milwaukee, WI, USA). The E. coli strains were grown at $37^{\circ} \mathrm{C}$ on Luria-Bertani (LB) plates or in LB broth. Antibiotics were used at the following concentrations when required, unless otherwise indicated: ampicillin $(100 \mu \mathrm{g} /$ $\mathrm{mL})$, chloramphenicol $(30 \mu \mathrm{g} / \mathrm{mL})$, erythromycin $(0.5 \mu \mathrm{g} /$ $\mathrm{mL})$, kanamycin $(50 \mu \mathrm{g} / \mathrm{mL})$, streptomycin $(50 \mu \mathrm{g} / \mathrm{mL})$, and cefoxitin $(5 \mu \mathrm{g} / \mathrm{mL})$.

\section{Construction of the mutant strain $\mathrm{Yb} 2 \Delta g / d M$ and complementation strain $\mathrm{cYb} 2 \Delta g / d M$}

The mutant strain $\mathrm{Yb} 2 \Delta g l d M$ was constructed by inserting the Tn4351 transposon into the AS87_08795 gene of the wild-type strain $\mathrm{Yb} 2$ (which was designated the AS87-RS08465 gene). Polymerase chain reaction (PCR) was used to identify the wild-type strain $\mathrm{Yb} 2$ and mutant strain $\mathrm{Yb} 2 \Delta g l d M$ with the primers $16 \mathrm{~S}$ rRNAF/16S rRNA-R and AS87_RS08465-F/AS87_RS08465-R, respectively (Table 1 ). Inverse PCR was used to determine the insertion site of the transposon in the mutant strain. Briefly, genomic DNA of the mutant strain was digested with HindIII and ligated to form a closed circle. DNA adjacent to the insertion site was amplified with the Tn4351-specific primers TN4351-F/TN4351-R. DNA sequencing data were compared to a database using a BLAST search at the National Center for Biotechnology Information website. The polar effect of the mutation was determined by qPCR analysis of the adjacent gene's expression.

The shuttle plasmid pCP29, which carries the ompA promoter of $R$. anatipestifer, was used to construct the complementation strain cYb2 $\Delta g l d M$ as described previously [26]. Briefly, the open reading frame (ORF) of $g l d M$ was amplified from $R$. anatipestifer $\mathrm{Yb} 2$ genomic DNA with the primers gldM-orf-F/gldM-orf-R, digested with $K p n \mathrm{I}$ and $\mathrm{XhoI}$, and ligated into pCP29 that had been digested with the same enzymes, generating the pCP29gld $M$ plasmid. The pCP29-gldM plasmid was then transferred into the mutant strain $\mathrm{Yb} 2 \Delta g l d M$ by conjugation to construct the complementation strain cYb2 $\Delta$ gld $M$.

\section{Protein expression and antibody production}

A 1464-bp fragment encoding the extramembranous part of GldM was amplified from the $R$. anatipestifer Yb2 genome with the primers GldM-pro-F/GldM-pro$\mathrm{R}$ and ligated into the pET-30a $(+)$ vector at the BamHI and SalI cloning sites. The resulting plasmid, pET-gldM, was sequenced and confirmed to be identical to the $R$. anatipestifer gldM sequence in the GenBank database (accession number AS87_RS08465). Competent E. coli strain BL21(DE3) cells were transformed with the recombinant plasmid $\mathrm{pET}-$ gld $M$ to express the protein. The transformed E. coli cells were cultured, and recombinant GldM protein (rGldM) expression was induced with $1 \mathrm{mM}$ isopropyl $\beta$-D-1-thiogalactopyranoside for $6 \mathrm{~h}$ at $37{ }^{\circ} \mathrm{C}$ with shaking. The cells were harvested by centrifugation at $10000 \times g$ for $5 \mathrm{~min}$ at $4{ }^{\circ} \mathrm{C}$, resuspended in lysis buffer (20 $\left.\mathrm{mM} \mathrm{Na}_{3} \mathrm{PO}_{4}, 0.5 \mathrm{M} \mathrm{NaCl}, \mathrm{pH} 7.4\right)$, and purified with HisTrap affinity columns (GE Healthcare, Uppsala, Sweden) according to the manufacturer's protocol. Aliquots of the fractions obtained were analyzed via sodium dodecyl sulfate-polyacrylamide gel electrophoresis (SDSPAGE). The protein concentrations were measured with a BCA protein assay kit (Beyotime, Shanghai, China), with bovine serum albumin (BSA) as the standard.

Two 2-month-old New Zealand rabbits were immunized three times with purified $R$. anatipestifer rGldM at 2-week intervals at a dose of $1 \mathrm{mg}$ of purified rGldM in the same volume of Montanide ISA $50 \mathrm{~V}$ adjuvant (SEPPIC, Paris, France). The preimmune and postimmune sera were tested with an indirect enzyme-linked immunosorbent assay (ELISA) for rGldM to confirm the presence of anti-GldM antibodies. Qualified antiserum was screened via Western blotting.

\section{Western blotting analysis Identification of $\mathrm{CYb2 \Delta g/dM}$ by Western blotting}

To determine whether the complementation strain cYb2 $\Delta g l d M$ expressed GldM, whole-cell proteins of the wild-type strain $\mathrm{Yb} 2$, mutant strain $\mathrm{Yb} 2 \Delta g l d M$, and complementation strain cYb2 $\Delta$ gld $M$ were extracted and separated by SDS-PAGE and then electrophoretically transferred onto nitrocellulose (NC) membranes (Millipore, Billerica, MA, USA). The membranes were blocked in phosphate-buffered saline (PBS) containing $5 \%$ nonfat milk, washed with PBS containing 0.05\% Tween 20, and incubated overnight with the rabbit anti-rGldM polyclonal antibody. A horseradish peroxidase-conjugated goat anti-rabbit IgG polyclonal antibody (Bio-Rad Laboratories, Hercules, CA, USA) was then applied, and the specific bands were developed with the Basic Luminol Chemiluminescent Kit (S-Wb001), visualized using a Tanon 5200 automatic chemiluminescence image analysis system (Tanon, Shanghai, China), and quantified using ImageJ software (National Institutes of Health, Rockville, USA). A rabbit anti-TonB-dependent receptor antibody was used as the control for protein loading. The intensities of the protein bands were analyzed with Quantity One software (Bio-Rad Laboratories).

\section{Subcellular localization of GIdM}

The cytoplasmic proteins and membrane proteins from the wild-type strain $\mathrm{Yb} 2$ were fractioned with a bacterial membrane protein extraction kit (BestBio, Shanghai, China) according to the manufacturer's protocol. The 
protein concentrations were determined with a BCA protein assay kit (Beyotime), with BSA as the standard. For Western blotting analysis, the subcellular fractions were separated by SDS-PAGE and then transferred onto a NC membrane. Western blotting was performed as described above. The rabbit anti-TonB-dependent receptor antibody was used as the control for protein loading.

\section{Gliding motility assay}

The $R$. anatipestifer wild-type strain $\mathrm{Yb} 2$, mutant strain $\mathrm{Yb} 2 \Delta g l d M$, and complementation strain $\mathrm{cYb} 2 \Delta g l d M$ were examined for movement over agar surfaces as previously described, with some modifications $[27,28]$. Briefly, each strain was grown on TSA plates for $12 \mathrm{~h}$, washed with TSB, and diluted to $2.5 \times 10^{3}$ colony forming units $(\mathrm{CFU}) / \mathrm{mL}$. Aliquots $(50 \mu \mathrm{L})$ of the cultures were plated onto TSB medium containing $0.5 \%$ agar. The colonies were grown for $24 \mathrm{~h}$ at $37{ }^{\circ} \mathrm{C}$ and examined by phasecontrast microscopy (Nikon D-Eclipse C1, Japan).

\section{Measurement of protease activity}

The proteolytic activity of each strain was quantified as described previously [29] with modifications. In brief, the strains $\mathrm{Yb} 2, \mathrm{Yb} 2 \Delta g l d M$, and $\mathrm{c} \mathrm{Yb} 2 \Delta$ gld $M$ were grown on TSA plates for $12 \mathrm{~h}$; washed with sterile PBS; and adjusted to $2.5 \times 10^{9} \mathrm{CFU} / \mathrm{mL}$. These strains were then used to inoculate $5 \mathrm{~mL}$ volumes of ADCF-mAb medium (Hyclone) and incubated for $8 \mathrm{~h}$ at $37{ }^{\circ} \mathrm{C}$ with shaking at $220 \mathrm{rpm}$. The cultures were centrifuged at $19950 \times g$ for $10 \mathrm{~min}$, and the supernatants were purified by passage through $0.45 \mu \mathrm{M}$ HT Tuffryn syringe filters (PALL Life Sciences, Ann Arbor, MI, USA). The bacterial pellets were dried in an $80{ }^{\circ} \mathrm{C}$ heat block for $3 \mathrm{~h}$, and the dry weights of the cell pellets were measured to calculate the proteolytic activity. A 2\% azocasein (Sigma) solution was prepared in $0.05 \mathrm{M}$ Tris- $\mathrm{HCl}$ (pH 7.4). The cell-free supernatant $(50 \mu \mathrm{L})$ was mixed with $50 \mu \mathrm{L}$ of azocasein substrate and incubated at $37^{\circ} \mathrm{C}$ for $3 \mathrm{~h}$. Triplicate assays were performed for each supernatant sample and negative controls (50 $\mu \mathrm{L}$ of ADCF-mAb medium). After incubation, $130 \mu \mathrm{L}$ of $10 \%$ trichloroacetic acid was added to each sample, mixed, allowed to stand for $10 \mathrm{~min}$ at room temperature, and centrifuged at $19950 \times g$ for $20 \mathrm{~min}$ to remove precipitated azocasein. An aliquot $(100 \mu \mathrm{L})$ of the soluble supernatant was added to a flat-bottomed 96-well plate, and $200 \mu \mathrm{L}$ of $1 \mathrm{M} \mathrm{NaOH}$ was added and mixed. The optical density at a wavelength of $450 \mathrm{~nm}\left(\mathrm{OD}_{450}\right)$ was determined with an iMark Microplate Absorbance Reader (Bio-Rad Laboratories). The raw optical density values at $450 \mathrm{~nm}\left(\mathrm{OD}_{450}\right)$ obtained from the triplicate assays were averaged for each supernatant sample, and the mean negative control $\mathrm{OD}_{450}$ was subtracted from these values. The proteolytic activity per milligram of dry cells was calculated as ([mean sample $\mathrm{OD}_{450}$ - mean negative control $\left.\left.\mathrm{OD}_{450}\right]\right) \times 1000 \times 100 /$ dry weight of the bacterial pellet.

\section{SDS-PAGE and liquid chromatography-tandem mass spectrometry (LC-MS/MS) analyses}

The $R$. anatipestifer wild-type strain $\mathrm{Yb} 2$, mutant strain $\mathrm{Yb} 2 \Delta g l d M$ and complementation strain cYb2 $\Delta g l d M$ were grown in $200 \mathrm{~mL}$ of $\mathrm{ADCF}$ medium at $37^{\circ} \mathrm{C}$ with shaking until $\mathrm{OD}_{600}$ reached 0.8 . The cultures were centrifuged at $8000 \times g$ for $10 \mathrm{~min}$, and the supernatants were purified by passage through $0.22 \mu \mathrm{m}$ polyvinylidene difluoride filters. The proteins in the supernatants were collected with 3-kDa Amicon Ultra Centrifugal Filter Units (Sigma) and stored at $-80{ }^{\circ} \mathrm{C}$ until analysis. The protein quality was determined by SDS-PAGE, followed by Coomassie Brilliant Blue staining. The proteins were analyzed with LCMS/MS as described previously [16]. The resulting MS/ MS data were evaluated using a MASCOT search engine based on the UniProt database. The sequences of identified proteins were searched using the BLAST server to identify homologous sequences of $R$. anatipestifer $\mathrm{Yb} 2$ and their putative functions.

\section{RNA sequencing and differential expression analysis}

Total RNA was isolated from the wild-type strain Yb2, mutant strain $\mathrm{Yb} 2 \Delta g l d M$ and complementation strain cYb2 $\Delta$ gldM with TRIzol Reagent (Invitrogen, Carlsbad, CA, USA) according to the manufacturer's instructions. Ribosomal RNA was removed with the Ribo-Zero Magnetic Gold Kit (Epicenter, USA), and Illumina RNA-Seq libraries were generated with the TruSeq SR Cluster Kit v3-cBot-HS (Illumina). The complete libraries were sequenced for 100 cycles on an Illumina HiSeq 2000 system according to the manufacturer's instructions [2].

Low-quality reads and adaptors were removed from raw reads. Cleaned reads were aligned to the $R$. anatipestifer $\mathrm{Yb} 2$ genome using the RNASequel software HISAT (Version 2.0.1-beta) [30, 31]. Trimmed reads were aligned to the $R$. anatipestifer $\mathrm{Yb} 2$ genome with the TopHat2 software (version 2.0.9) [32]. The transcript levels were calculated as fragments per kilobase of cDNA per million fragments mapped. Differentially expressed genes with a fold change (cutoff) of 2.0 were detected with the Cufflinks software (version 2.1.1) [33] and were considered statistically significant if the fold change was $>2.0$ and the $p$ value was $<0.05$.

\section{Real-time quantitative PCR (qPCR) analysis}

qPCR was used to confirm the transcription levels of the differentially expressed genes identified in the RNA-Seq analysis. Gene-specific primers were designed with the Primer3 online software version 0.4 .0 and are described 
in Additional file 1. The gene encoding L-lactate dehydrogenase $(l d h)$ was used as the internal control, and its transcription was measured with the primers RA ldh-F/RA ldh-R (Table 1) [34, 35]. The RNA samples were extracted and purified as described above. cDNA was synthesized with PrimeScript RT Master Mix (Takara) according to the manufacturer's protocol and diluted threefold as the template. qPCR was performed with GoTaq qPCR Master Mix (Promega, Fitchburg, WI, USA) using the following parameters: $95^{\circ} \mathrm{C}$ for $2 \mathrm{~min}, 40$ cycles of $95^{\circ} \mathrm{C}$ for $15 \mathrm{~s}$, $55^{\circ} \mathrm{C}$ for $15 \mathrm{~s}$ and $68{ }^{\circ} \mathrm{C}$ for $20 \mathrm{~s}$, followed by one cycle of $95{ }^{\circ} \mathrm{C}$ for $15 \mathrm{~s}, 60{ }^{\circ} \mathrm{C}$ for $15 \mathrm{~s}$ and $95^{\circ} \mathrm{C}$ for $15 \mathrm{~s}$. The reactions for each sample were performed in triplicate and run on a Mastercycler ep realplex4 apparatus (Eppendorf, Germany). The transcription levels were quantified with the $2^{-\Delta \Delta \mathrm{Ct}}$ method [33].

\section{Bacterial adherence and invasion assays}

Adhesion and invasion assays were performed on Vero cells (ATCC CCL-81) as previously described [2]. Briefly, Vero cells (approximately $2.5 \times 10^{5}$ cells/well) were infected with the appropriate strain at a multiplicity of infection (MOI) of 50, incubated for $1.5 \mathrm{~h}$ at $37{ }^{\circ} \mathrm{C}$ under $5 \% \mathrm{CO}_{2}$, rinsed three times with sterile PBS, and lysed with $0.1 \%$ trypsin $(100 \mu \mathrm{L} /$ well). The number of celladherent bacteria was determined after each lysate was serially diluted tenfold and spread onto a TSA plate. For the invasion assay, infected cells were incubated for an additional $1 \mathrm{~h}$ with Dulbecco's modified Eagle's medium supplemented with $100 \mu \mathrm{g} / \mathrm{mL}$ gentamicin to kill any extracellular bacteria. Infected cells were then washed three times with PBS and lysed, and the number of intracellular bacteria was determined. All the assays described here were performed in triplicate and replicated three times.

\section{Assessment of virulence in vivo}

The median lethal dose $\left(\mathrm{LD}_{50}\right)$ of the mutant strain $\mathrm{Yb} 2 \Delta g l d M$ was determined as described in our previous study [25]. To determine whether $R$. anatipestifer gldM is involved in the systemic invasion and dissemination of the bacterium, the bacterial loads in the blood of infected ducks were measured and analyzed. Briefly, 30 18-day-old Cherry Valley ducks were randomly divided into three groups (10 ducks per group) and intramuscularly infected with the wild-type strain $\mathrm{Yb2}$, mutant strain $\mathrm{Yb} 2 \Delta g l d M$, or complementation strain $\mathrm{cYb} 2 \Delta g l d M$ at a dose of $10^{6} \mathrm{CFU}$ in $0.5 \mathrm{~mL}$ of PBS. Blood samples were collected at 6,12 , and $24 \mathrm{~h}$ post-infection (hpi), diluted appropriately, and plated on TSA for bacterial counting [2].

\section{Statistical analysis}

Statistical analyses were conducted using GraphPad Software version 6.0 (La Jolla, CA, USA). One-way analysis of variance (ANOVA) was used for analyses of growth curves, protease activity, adhesion and invasion data, and two-tailed independent Student's $t$ test was used for analyses of the bacterial loads in blood. $p$ values of $<0.05$ were considered significant.

\section{Results}

Characterization of the mutant strain Yb2 $\Delta g / d M$ and complementation strain $\mathrm{CYb} 2 \Delta$ gldM

The mutant strain $\mathrm{Yb} 2 \Delta g l d M$, in which the $\mathrm{Tn} 4351$ transposon was inserted at nucleotide position $1307 \mathrm{bp}$ of the AS87_RS08465 gene, was identified in our previous study [25]. PCR amplification using the primers $16 \mathrm{~S}$ rRNA-F/16S rRNA-R and RA_08465-F/RA_08465-R produced a 792-bp fragment of $16 \mathrm{~S}$ rRNA and a 1560 bp fragment of $g l d M$, respectively, in the wild-type strain Yb2 (lane 1) and complementation strain cYb2 $\Delta$ gld $M$ (lane 3), whereas no 1560-bp fragment was amplified from the mutant strain $\mathrm{Yb} 2 \Delta$ gld $M$ because of the insertion (Figure 1A). No polar effect of the gldM mutation was found by qPCR analyses on the adjacent genes (Figure 1B). The AS87_RS08465 gene is $1560 \mathrm{bp}$ in length and encodes a predicted 520 -amino-acid GldM protein. A phylogenetic tree showed that $R$. anatipestifer and many other members of the phylum Bacteroidetes encode this protein [12]. GldM was originally identified as a protein required for bacterial gliding motility $[22,36]$, and a further study confirmed that GldM is a component of T9SS and involved in protein secretion [23, 37]. The growth rates of the wild-type strain $\mathrm{Yb} 2$, mutant strain $\mathrm{Yb} 2 \mathrm{gld} M$, and complementation strain cYb2gld $M$ in TSB medium did not significantly differ (Figure 1C).

\section{Identification and localization of GldM}

Histidine-tagged rGldM was efficiently expressed in E. coli BL21(DE3). As shown in Figure 2A, E. coli BL21(DE3) transformed with pET-30a $(+)$ displayed no GldM (57-kDa) band (lane 1). The presence of a $57-\mathrm{kDa}$ band was indicated in lanes 2 and 3 of a Coomassie bluestained SDS-PAGE gel in which E. coli BL21(DE3) transformed with pET-gldM and its supernatant were loaded, respectively. After purification with HisTrap affinity columns, rGldM was identified as a single band in the gel (lane 4).

The expression of GldM in strains $\mathrm{Yb} 2, \mathrm{Yb} 2 \Delta g l d M$, and cYb2 $\Delta g l d M$ was determined by Western blotting. As shown in Figure 2B, a $57-\mathrm{kDa}$ band was detected in the wild-type strain $\mathrm{Yb} 2$ (lane 1) and complementation strain cYb2 2 gld $M$ (lane 3) but was absent in the mutant strain 


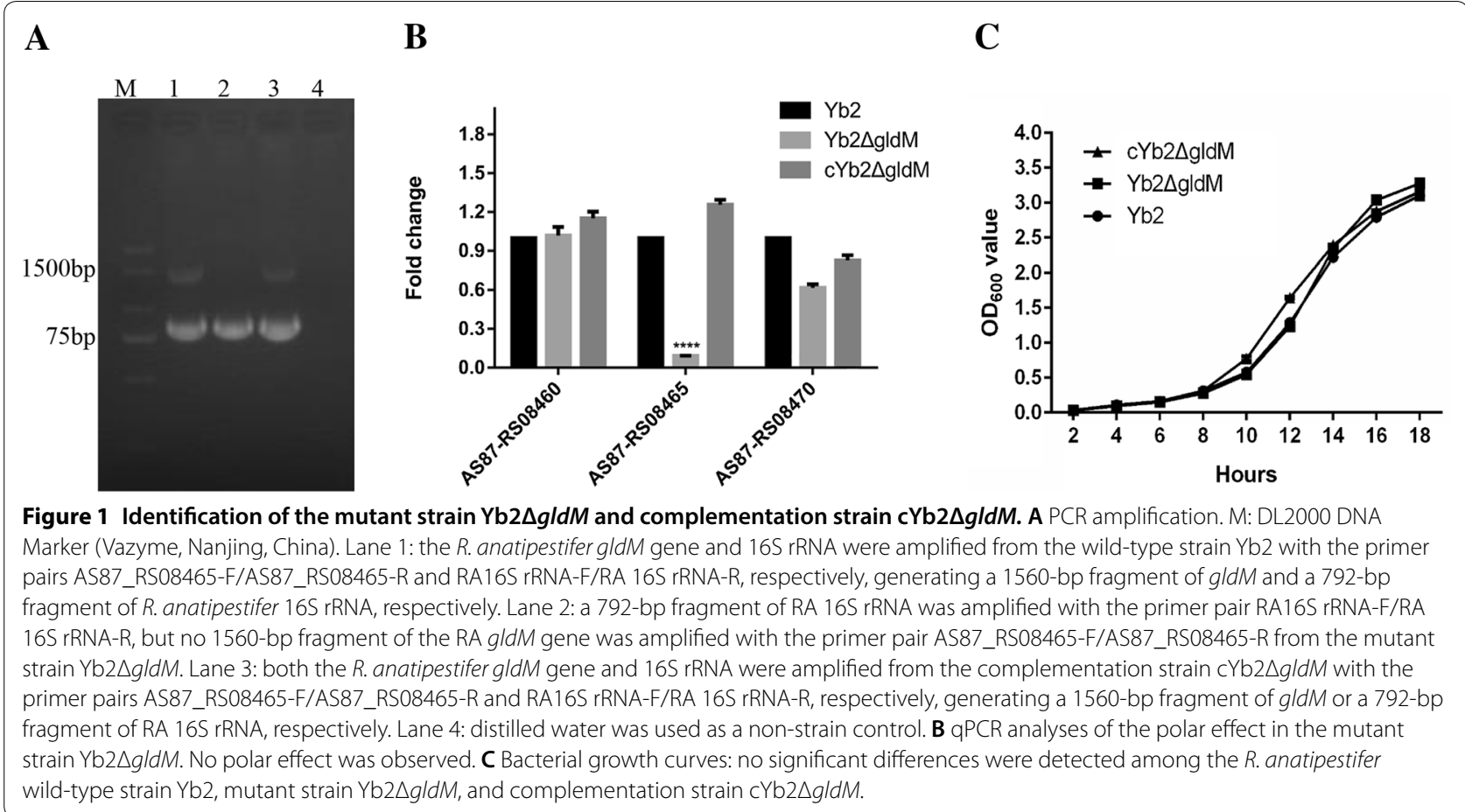

A

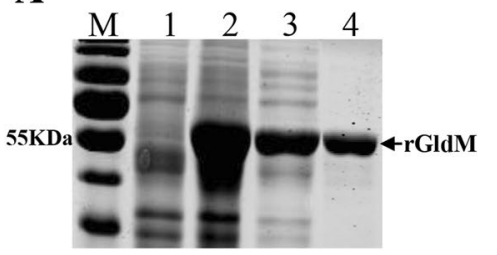

B

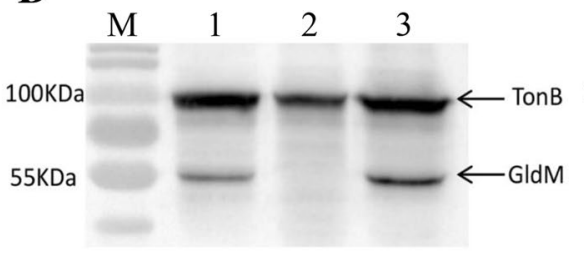

C

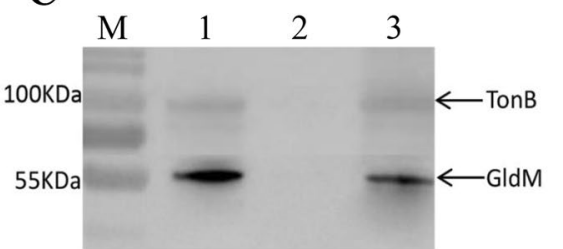

Figure 2 Expression and identification of $\boldsymbol{R}$. anatipestifer GIdM. A SDS-PAGE analysis of recombinant GldM (rGldM) expression. Lane M: PageRuler Prestained Protein Ladder (Thermo Scientific, Waltham, MA, USA); lane 1: E. coli BL21(DE3) transformed with pET-30a(+), with IPTG induction; lane 2: E. coli BL21(DE3) transformed with pET-gldM, with IPTG induction; lane 3: supernatant from E. coli BL21(DE3) transformed with pET-gldM, with IPTG induction; lane 4: purified rGIdM. B Identification of the complementation strain cYb2gldM using a Western blotting assay. Lane 1: whole-cell proteins from the wild-type strain Yb2; lane 2: whole-cell proteins from the mutant strain Yb2 $\Delta$ gldM; lane 3: whole-cell proteins from the complementation strain cYb2 $\triangle$ gldM. C Subcellular localization of GldM in R. anatipestifer using a Western blotting assay. Lane 1: whole-cell proteins from the wild-type strain Yb2; lane 2: extract of cytoplasmic proteins from Yb2; lane 3: extract of membrane proteins from Yb2. A second antibody directed against the TonB-dependent receptor of $R$. anatipestifer was used to control for protein loading in both assays.

$\mathrm{Yb} 2 \Delta g l d M$ (lane 2), indicating that GldM is encoded by the AS87_RS08465 gene and that the expression of GldM is rescued in the complementation strain cYb2gldM. This analysis clearly indicated that the AS87_RS08465 gene was successfully disrupted in the mutant strain $\mathrm{Yb} 2 \Delta$ gldM.

To determine the subcellular location of GldM in $R$. anatipestifer, the cytoplasmic and membrane fractions of bacterial cells were extracted and analyzed by Western blotting. As shown in Figure $2 \mathrm{C}$, a single $57-\mathrm{kDa}$ band corresponding to GldM was detected in whole-cell proteins from the wild-type strain Yb2 (lane 1, positive control) and purified cytomembrane fraction (lane 3). No band was found in cytoplasmic proteins from Yb2 (lane 2 ), indicating that GldM is expressed in the cytomembrane of $R$. anatipestifer.

\section{Riemerella anatipestifer mutant strain Yb2 $\Delta g / d M$ is defective in gliding motility}

A transposon insertion in $g l d M$ resulted in the complete loss of F. johnsoniae motility, as reported previously [22]. In our study, the motility phenotypes of the wild-type 
strain $\mathrm{Yb} 2$, mutant strain $\mathrm{Yb} 2 \Delta$ gld $M$, and complementation strain $\mathrm{cYb} 2 \Delta g l d M$ were examined. When cultured on $0.5 \%$ TSA plates, the wild-type strain $\mathrm{Yb} 2$ formed spreading colonies, whereas the mutant strain $\mathrm{Yb} 2 \Delta g l d M$ formed nonspreading colonies (Figures $3 \mathrm{~A}$ and $\mathrm{B}$ ). The gliding motility of the $R$. anatipestifer complementation strain $\mathrm{cYb} 2 \Delta g l d M$ on the agar surface was restored (Figure 3C).

\section{Mutant strain Yb2 $\Delta g / d M$ displays defective protein secretion}

T9SS is involved in the secretion of many proteins, such as the soluble extracellular chitinase ChiA, in the environmental bacterium $F$. johnsoniae $[16,38]$ and in the secretion of gingipain proteases and cell-surface adhesins in Porphyromonas gingivalis, which have been confirmed to be virulence factors in periodontitis [14, 23]. Cell-free culture fluids of the wild-type strain $\mathrm{Yb} 2$, mutant strain
$\mathrm{Yb} 2 \Delta g l d M$ and complementation strain cYb2 $\Delta g l d M$ were collected and examined with SDS-PAGE to detect any differences in secreted proteins. We found fewer protein bands or smaller quantities of protein in the culture fluid from the mutant strain $\mathrm{Yb} 2 \Delta g l d M$ than from that of the wild-type strain $\mathrm{Yb} 2$ and complementation strain $\mathrm{cYb} 2 \Delta g l d M$ (Figure 4A).

The proteins in the cell-free culture fluids of strains $\mathrm{Yb} 2, \mathrm{Yb} 2 \Delta g l d M$ and $\mathrm{cYb} 2 \Delta g l d M$ were identified with LC-MS/MS. Our results indicate that the mutant strain $\mathrm{Yb} 2 \Delta g l d M$ was defective in the digestion of proteins (Figure 4B), consistent with the fact that the enzymes involved in the digestion of these polymers are secreted by T9SS. In addition, 165 proteins were differentially identified in the cell-free culture fluids of $\mathrm{Yb} 2 \Delta g l d M$ compared to those of $\mathrm{Yb} 2$ (Additional file 2). Among these proteins, nine had typical T9SS CTDs (Table 2) and could be predicted as T9SS proteins. The other proteins
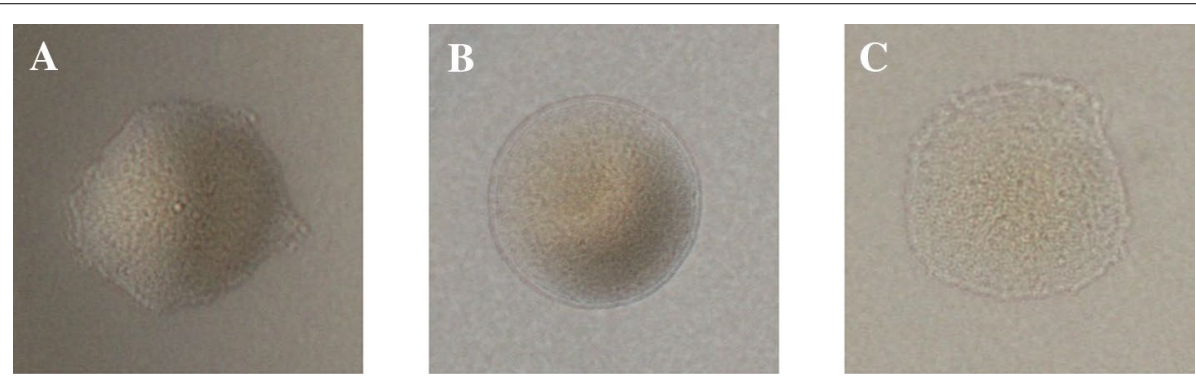

Figure 3 Photomicrographs of R. anatipestifer colonies. Colonies were grown for $24 \mathrm{~h}$ at $37^{\circ} \mathrm{C}$ on TSA plates. Photomicrographs were taken with a Photometrics CoolSNAP ${ }_{\text {ff }}^{2}$ camera mounted on a phase-contrast microscope. A Wild-type R. anatipestifer Yb2. B Mutant strain Yb2 $\triangle g / d M$. C Complementation strain Yb2 $\Delta g / d M$.

A

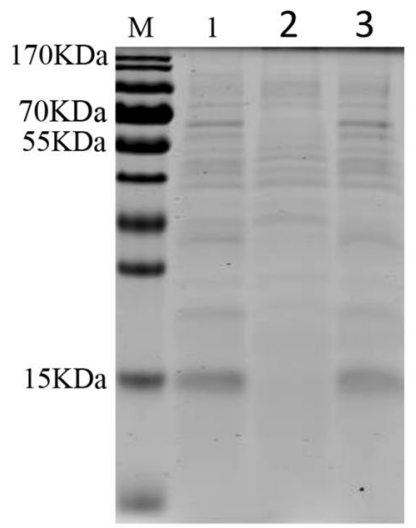

B

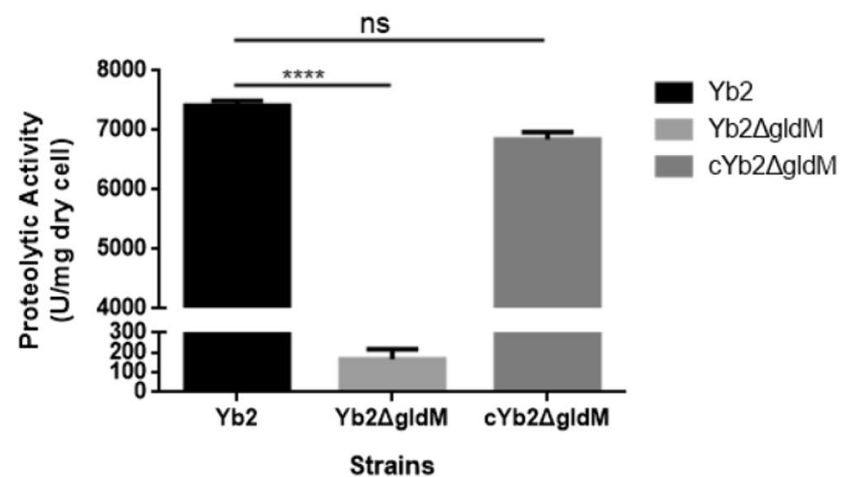

Figure 4 Analysis of secreted proteins. A SDS-PAGE profile of extracellular proteins. Lane 1: wild-type strain Yb2; lane 2: mutant strain Yb2 $\Delta$ gldM; lane 3: complementation strain $\mathrm{cYb} 2 \Delta g / d M$. Strains were incubated with shaking until cultures reached an $\mathrm{OD}_{600}$ of 0.8 . The same volume of concentrated cell-free supernatant from each strain was separated by SDS-PAGE, and proteins were visualized with Coomassie Brilliant Blue staining. B Determination of proteolytic activity. The error bars represent the standard deviation calculated from three independent experiments performed in triplicate $\left.{ }^{* * * *} p<0.0001 ; n s, p>0.05\right)$ 
Table 2 Predicted R. anatipestifer Yb2 proteins secreted by T9SS identified with LC-MS/MS

\begin{tabular}{|c|c|c|c|c|c|c|}
\hline Locus tag & $\begin{array}{l}\text { Molecular } \\
\text { mass }\left(K^{a}\right)\end{array}$ & T9SS CTD & Proteins & Predicted protein function & $\begin{array}{l}\text { Relative } \\
\text { change } \\
\text { of proteinc }\end{array}$ & $\begin{array}{l}\text { Relative } \\
\text { change } \\
\text { of protein }{ }^{d}\end{array}$ \\
\hline AS87_RS03090 & 13.49 & + & Uncharacterized protein & Unknown & 6.0762 & 1.6765 \\
\hline AS87_RS06600 & 117.29 & + & Pkd domain containing protein & Collagenolytic of the catalytic & 0.1004 & 1.1848 \\
\hline AS87_RS07295 & 93.945 & + & Fibronectin type iii domain protein & Serine-type endopeptidase activity & 0.0274 & 0.9055 \\
\hline AS87_RS02625 & 39.812 & + & Endonuclease i & Endonuclease activity & 0.2592 & 0.7272 \\
\hline AS87_RS00980 & 68.225 & + & Metallophosphoesterase (MPPE) & Acid phosphatase activity & 0.0434 & 1.3085 \\
\hline AS87_RS04190 & 77.52 & + & Subtilisin-like serine protease & Serine-type endopeptidase activity & 0.2041 & 1.1190 \\
\hline AS87_RS03200 & 117.12 & + & Uncharacterized protein & Unknown & 0.0063 & 1.2016 \\
\hline AS87_RS02020 & 83.131 & + & $\begin{array}{l}\text { Immunoreactive } 84 \text { kDa antigen } \\
\text { pg93 }\end{array}$ & Metal ion binding & 0.3273 & 1.1742 \\
\hline AS87_RS00835 & 161.5 & + & $\begin{array}{l}\text { Peptidase s8 and s53 subtilisin } \\
\text { kexinsedolisin }\end{array}$ & Serine-type endopeptidase activity & 0.0045 & 1.2558 \\
\hline
\end{tabular}

may have had novel targeting sequences or were released by an indirect process that involved T9SS. The complementation strain $\mathrm{cYb} 2 \Delta$ gld $M$ recovered most of the proteins secreted by the mutant strain $\mathrm{Yb} 2 \Delta g l d M$, and only 45 proteins were differentially expressed between $\mathrm{cYb} 2 \Delta g l d M$ and $\mathrm{Yb} 2$ (Additional file 3). Moreover, the nine secreted proteins with the typical T9SS CTDs were all recovered in the complementation strain cYb2 $\Delta$ gld $M$ (Table 2).

\section{gldM disruption affects gene expression}

Strand-specific Illumina RNA-Seq analysis was used to investigate the differentially expressed genes among the wild-type strain $\mathrm{Yb} 2$, mutant strain $\mathrm{Yb} 2 \Delta$ gld $M$ and complementation strain cYb2 $\Delta g l d M$. In total, 15 genes were upregulated or downregulated in the mutant strain $\mathrm{Yb} 2 \Delta g l d M$ relative to their expression in the wildtype strain $\mathrm{Yb} 2$. qPCR confirmed that six genes in the mutant strain $\mathrm{Yb} 2 \Delta g l d M$ were upregulated $>2$-fold at the transcription level (Table 3). The AS87_RS00230 gene encodes the protein identified as SprT, which is a component of T9SS. The AS87_RS09480 gene encodes a protein involved in "pilus twitching motility". AS87_ RSO8665 encodes a protein annotated as the Tat pathway signal sequence domain-containing protein. The AS87 RS02915, AS87_RS09140, and AS87_RS02755 genes encode hypothetical proteins. Compared with the wildtype strain $\mathrm{Yb} 2$, five genes were downregulated $>2$-fold in the mutant strain Yb2 2 gldM. The AS87_RSO1360 and AS87_RS01350 genes, respectively, encode the hemolysin $\mathrm{D}$ protein and a CzcA family member, which is a heavy metal efflux pump. The putative product of the AS87 RS01355 gene is a transporter. Both the AS87_RS07850 and AS87_RS01365 genes encode hypothetical proteins. The differentially expressed genes in the mutant strain $\mathrm{Yb} 2 \Delta g l d M$ were all restored in the complementation strain cYb2 $\Delta$ gld $M$ (Table 3 ). The gliding motility protein SprT is a component of T9SS and is involved in protein secretion and cell movement. The transporter encoded by $A S 87 \_R S 01355$ is also involved in protein secretion. These results suggest that AS87_RS08465 regulates genes that are mainly responsible for protein secretion and gliding motility in $R$. anatipestifer.

\section{Deletion of $g / d M$ reduces bacterial adherence and invasion capacities}

The bacterial adherence and invasion capacities of the wild-type strain $\mathrm{Yb} 2$, mutant strain $\mathrm{Yb} 2 \Delta g l d M$, and complementation strain cYb2 $\Delta g l d M$ were determined in Vero cells. When cells were infected with $R$. anatipestifer at a $\mathrm{MOI}$ of 50, the number of cell-adherent $\mathrm{Yb} 2 \Delta$ gld $M$ bacteria was $1.69 \times 10^{4} \mathrm{CFU} /$ well, which was significantly lower than the numbers of Yb2 $\left(6.54 \times 10^{4} \mathrm{CFU} /\right.$ well $)$ and cYb2 $\Delta$ gldM $\left(7.59 \times 10^{4} \mathrm{CFU} /\right.$ well $)$ cell-adherent bacteria. When the cell-invasive bacteria were counted, the number of $\mathrm{Yb} 2 \Delta g l d M$ was $5.24 \times 10^{3} \mathrm{CFU} /$ well, almost threefold lower than the number of Yb2 $\left(1.79 \times 10^{4} \mathrm{CFU} /\right.$ well) or cYb2 $\Delta g l d M\left(2.40 \times 10^{4} \mathrm{CFU} /\right.$ well). These results demonstrate that mutation of the $R$. anatipestifer gldM gene significantly reduces bacterial adherence and invasion (Figures $5 \mathrm{~A}$ and $\mathrm{B}$ ). 
Table 3 qPCR verification of differentially expressed genes in the mutant strain Yb2 $\Delta g / d M$ and complementation strain cYb2 $\Delta g / d M$

\begin{tabular}{|c|c|c|c|}
\hline \multirow[t]{2}{*}{ Gene locus $^{a}$} & \multirow[t]{2}{*}{ Description of genes } & \multicolumn{2}{|l|}{$2^{-\Delta \Delta C t}$} \\
\hline & & $\mathrm{Yb} 2 \Delta g / d M$ & $\mathrm{cYb} 2 \Delta g / d N$ \\
\hline AS87_RS00230 b & PorT protein & 3.5925 & 1.472 \\
\hline AS87_RS02915 & Hypothetical protein & 2.445 & 1.3755 \\
\hline AS87_RS09140 & Hypothetical protein & 2.0875 & 1.2058 \\
\hline AS87_RS09480 & Twitching motility protein PilT & 2.06 & 1.2397 \\
\hline AS87_RS02755 & Hypothetical protein & 2.0533 & 0.9432 \\
\hline AS87_RS08665 & Tat pathway signal sequence domain-containing protein & 2.03 & 1.3947 \\
\hline AS87_RS09585 & Hypothetical protein & 1.6533 & 1.1028 \\
\hline AS87_RS08925 & SusC/RagA family TonB-liked outer membrane protein & 1.4225 & 1.6531 \\
\hline AS87_RS08050 & Carbohydrate-bonding protein & 1.3875 & 0.8593 \\
\hline AS87_RS04485 & Cbb3-type cytochrome oxidase component FixQ & 1.35 & 1.021 \\
\hline AS87_RS07185 & Hypothetical protein & 1.28 & 1.0352 \\
\hline AS87_RS09360 & Hypothetical protein & 1.2725 & 1.1408 \\
\hline AS87_RS05780 & Hypothetical protein & 1.12 & 0.8526 \\
\hline AS87_RS05060 & Hypothetical protein & 1.0733 & 0.7474 \\
\hline AS87_RS10475 & Hypothetical protein & 1.0233 & 0.8745 \\
\hline AS87_RS08045 & RagA family TonB-liked outer membrane protein & 0.985 & 1.2256 \\
\hline AS87_RS00555 & RNA polymerase sigma24 factor & 0.98 & 1.0867 \\
\hline AS87_RS08885 & Hypothetical protein & 0.947 & 1.591 \\
\hline AS87_RS09380 & Hypothetical protein & 0.945 & 1.0497 \\
\hline AS87_RS01535 & Hypothetical protein & 0.93 & 1.2226 \\
\hline AS87_RS05020 & Hypothetical protein & 0.9175 & 0.895 \\
\hline AS87_RS09995 & Hypothetical protein & 0.8825 & 1.4948 \\
\hline AS87_RS08585 & $\mathrm{ABC}$ transporter & 0.8425 & 1.5157 \\
\hline AS87_RS00880 & $\mathrm{ABC}$ transporter related protein & 0.6175 & 0.9659 \\
\hline AS87_RS08470 b & Gliding motility protein GldN & 0.615 & 0.7813 \\
\hline AS87_RS07850 & Hypothetical protein & 0.47 & 0.9075 \\
\hline AS87_RS01360 & Hemolysin D & 0.34 & 0.5586 \\
\hline AS87_RS01365 & Hypothetical protein & 0.33 & 0.8645 \\
\hline AS87_RS01350 & Heavy metal efflux pump, CzcA family & 0.295 & 0.7846 \\
\hline AS87_RS01355 & Transporter & 0.225 & 0.6925 \\
\hline
\end{tabular}

a Based on the $R$. anatipestifer Yb2 genome (accession number: CP007204).

b These genes encode T9SS components.

Mutation of the gldM gene attenuates bacterial virulence In our previous study, the $\mathrm{LD}_{50}$ of the mutant strain Yb2 $\operatorname{gld} M$ was shown to be $1.97 \times 10^{7} \mathrm{CFU}$, so its virulence was attenuated 184-fold compared with that of the wild-type strain $\mathrm{Yb} 2\left(1.07 \times 10^{5} \mathrm{CFU}\right)$ [25]. In this study, the bacterial loads in the blood of infected ducks were quantified to investigate the role of the AS87 $R S 08465$ gene in systemic infection in vivo. The bacterial loads of the mutant strain $\mathrm{Yb} 2 \Delta$ gld $M$ in infected duck's blood were $5.45 \times 10^{2} \mathrm{CFU} / \mathrm{mL}, 3.12 \times 10^{2} \mathrm{CFU} /$ $\mathrm{mL}$, and $3.2 \times 10^{2} \mathrm{CFU} / \mathrm{mL}$ at 12,24 , and $36 \mathrm{hpi}$, respectively, whereas the bacterial loads of the wild-type strain were $3.26 \times 10^{4} \mathrm{CFU} / \mathrm{mL}, 4.9 \times 10^{4} \mathrm{CFU} / \mathrm{mL}$, and
$2.18 \times 10^{6} \mathrm{CFU} / \mathrm{mL}$ at 12,24 , and $36 \mathrm{hpi}$, respectively (Figure 6), indicating that deletion of the gldM gene significantly attenuated the virulence of $R$. anatipestifer. Bacterial recovery from the blood of infected ducks was similar after infection with the complementation strain cYb2 $\Delta g l d M$ and wild-type strain Yb2.

\section{Discussion}

Genomic analyses have shown that T9SSs, also known as the "Por secretion system", are common in members of the phylum Bacteroidetes, including F. johnsoniae, P. gingivalis, Tannerella forsythia, and R. anatipestifer [12]. This novel protein secretion system, which has 

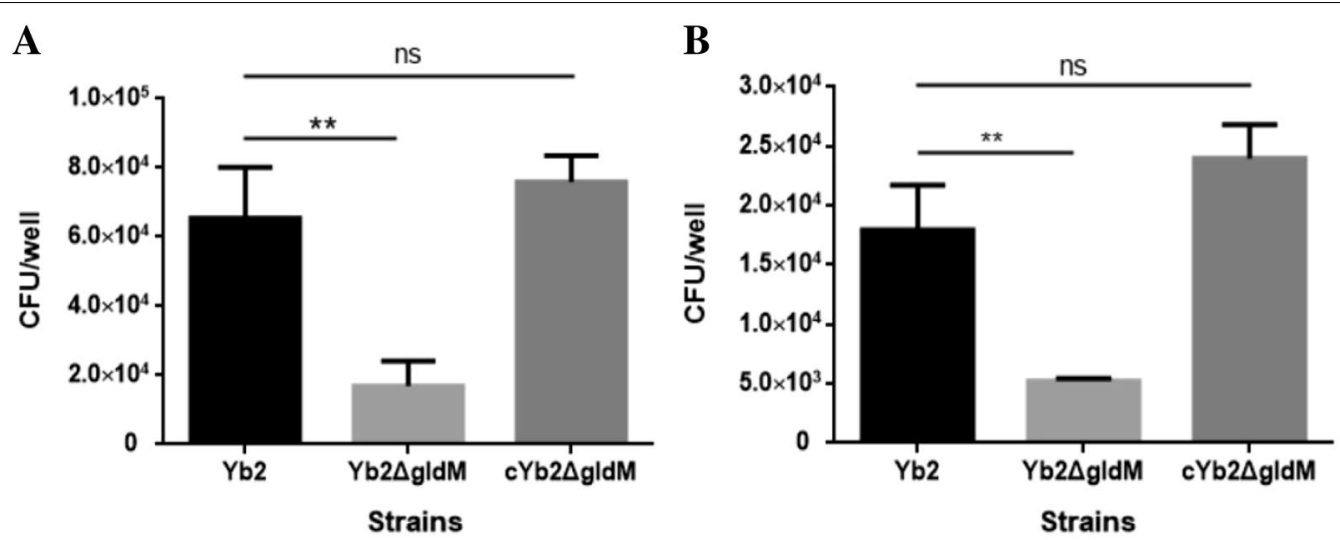

Figure 5 Bacterial adherence (A) and invasion (B) assays. The assays were performed on Vero cells, and the cells were infected with each strain at a $\mathrm{MOI}$ of 50. The data represent the counts of bacteria bound to or having invaded Vero cells in each well of a 24-well plate. The data are presented as the means \pm standard deviations of three independent experiments $\left({ }^{* *} p<0.01 ; n s, p>0.05\right)$.

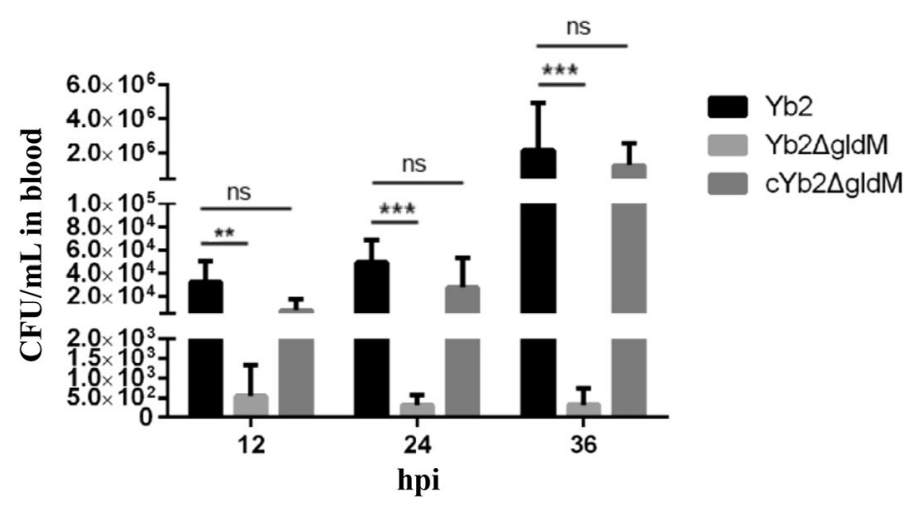

Figure 6 Determination of bacterial loads. Bacterial loads in the blood of ducks infected with R. anatipestifer Yb2, Yb2 $\Delta$ gldM, or cYb2 $\Delta$ gldM were determined at 12,24, and 36 hpi. Bacterial colony-forming units were counted and analyzed. The data are presented as the means \pm standard deviations of ten infected ducks and were analyzed using two-tailed independent Student's $t$ test. Asterisks indicate statistically significant differences between groups $\left({ }^{* *} p<0.01 ;{ }^{* *} p<0.001 ; \mathrm{ns}, p>0.05\right)$.

only recently been recognized and studied, functions in bacterial gliding motility and protein secretion [23, 39, 40]. In F. columnare, proteases and chondroitin sulfate lyases, which are known virulence factors, are secreted via T9SS [41]. In P. gingivalis, Arg-gingipain, Lys-gingipain, and Skp protein, which are virulence factors that cause human tissue damage, are also secreted by T9SS $[14,42,43]$.

GldM is a known cytoplasmic membrane component of the F. johnsoniae T9SS [22]. Therefore, GldM is potentially involved in harvesting cellular energy to power secretion and deliver motility adhesions [19]. Braun demonstrated that GldM is required for gliding in F. johnsoniae [22]. In the present study, Western blotting analysis showed that $R$. anatipestifer GldM was localized to the cytomembrane and that the mutant strain $\mathrm{Yb} 2 \Delta$ gldM was defective in gliding motility, forming nonspreading colonies on the surface of agar.
These results indicate that GldM is a membrane protein required for gliding in $R$. anatipestifer.

In F. johnsoniae, gldK, gldL, gldM, and gldN clustered together in the genome, and their products (GldK, GldL, GldM, and GldN, respectively) may form a complex [36]. In this study, qPCR revealed that the disruption of gldM dramatically downregulated the expression of gldN. The expression of the genes encoding a transporter (AS87_ RS01355), heavy metal efflux pump (AS87_RS01350), hemolysin D (AS87_RS01360) and two hypothetical proteins (AS87_RS01365 and AS87_RS07850) was also downregulated in the gldM deletion mutant strain $\mathrm{Yb} 2 \Delta g l d M$. These results suggest that $g l d M$ is associated with the expression of several genes that may work together in protein secretion. Several reports have demonstrated relationships between SprT and other T9SS components, and SprT is also required for the secretion of SprB, RemA, and chitinase, which function like 
GldM in $F$. johnsoniae, where gldK, gldM, gld $N$ and porT are components of T9SS and are associated with bacterial gliding motility [19]. Our results also showed that the expression of $\operatorname{spr} T$ (an orthologue of port in P. gingivalis) was significantly elevated in the mutant strain $\mathrm{Yb} 2 \Delta g l d M$. In addition, our LC-MS results showed that many secretion proteins were present at higher levels in the cell-free culture of $\mathrm{Yb} 2 \Delta g l d M$ than in $\mathrm{Yb} 2$, which may be related to the upregulation of the $\operatorname{spr} T$ gene, and the change in SprT secretion may compensate for the gldM deletion. Future studies are required to clarify the relationships of these proteins in various processes.

The $\operatorname{spr} T$ gene encodes the T9SS component SprT, which is involved in the protein secretion and virulence of $R$. anatipestifer [21]. However, the functions of other T9SS components in $R$. anatipestifer are still unclear. In this study, deletion of the $R$. anatipestifer gldM gene, which encodes a component of T9SS, significantly reduced the bacterial adherence and invasion capacities and the bacterial loads in infected duck blood compared with those of the wild-type strain Yb2. These findings provide powerful evidence that T9SS plays an important role in the pathogenicity of $R$. anatipestifer.

The SDS-PAGE profiles of cell-free cultures revealed that the levels of many secreted proteins were reduced or that these proteins were absent when gldM was mutated. LC-MS/MS demonstrated that more than 100 proteins were differentially secreted from the mutant strain $\mathrm{Yb} 2 \Delta$ gld $M$ compared to the wild-type strain $\mathrm{Yb} 2$. Genomic analysis showed that nine of the differentially expressed proteins had CTDs that were predicted to target them for secretion by T9SS. All of the CTDs belong to the TIGRFAM protein domain family TIGR04183 (and are referred to as "type A CTDs") [41], a conserved carboxyterminal domain of the extracellular proteinase family.

In summary, we showed that the $R$. anatipestifer AS87_ RS08465 gene encodes GldM, which is required for gliding motility, protein secretion, and bacterial virulence of the bacterium. More than 100 proteins were differentially secreted from the mutant strain $\mathrm{Yb} 2 \Delta g l d M$, suggesting their importance in the full virulence of $R$. anatipestifer. The molecular mechanisms by which these proteins act in bacterial virulence require further clarification, which should facilitate the development of effective strategies to control $R$. anatipestifer infection.

\section{Additional files}

Additional file 1. Primers used for real-time PCR analysis.
Additional file 2. Differentially secreted proteins of the wild-type strain $\mathrm{Yb} 2$ and mutant strain $\mathrm{Yb} 2 \Delta$ gldM.

Additional file 3. Differentially secreted proteins of the wild-type strain $\mathrm{Yb2}$ and complementation strain $\mathrm{CYb} 2 \Delta$ gldM.

\section{Abbreviations}

T9SS: type IX secretion system; CTDs: C-terminal domains; TSB: tryptic soy broth medium; TSA: tryptic soy agar; ELISA: enzyme-linked immunosorbent assay; PCR: polymerase chain reaction; CFU: colony forming unit; SDS-PAGE: sodium dodecyl sulfate polyacrylamide gel electrophoresis; $\mathrm{LD}_{50}$ : median lethal doses.

\section{Acknowledgements}

We thank the staff of Shanghai Applied Protein Technology Co. Ltd for LC-MS/ MS analysis.

\section{Authors' contributions}

$\mathrm{ZC}, \mathrm{XW}$ and $\mathrm{XR}$ performed the experiments, analyzed the data and prepared the manuscript. WH, KKM, and CD contributed reagents, materials and analysis tools. SY designed the study and revised the manuscript. All authors read and approved the final manuscript.

\section{Funding}

This work was supported by National Key R\&D Program (2016YFD0500805), National Natural Science Foundation of China (31602069), the Shanghai Key Project on Agricultural Development through Science and Technology (2016HNG4-1) and Co-innovation of Science and Technology Innovation Project in Chinese Academy of Agricultural Sciences (CAAS-XTCX2016011-04-8).

\section{Competing interests}

The authors declare that they have no competing interests.

\section{Author details}

${ }^{1}$ Shanghai Veterinary Research Institute, Chinese Academy of Agricultural Sciences (CAAS), Shanghai, China. ${ }^{2}$ Jiangsu Agri-animal Husbandry Vocational College, Veterinary Bio-pharmaceutical, Jiangsu Key Laboratory for High-

Tech Research and Development of Veterinary Biopharmaceuticals, Taizhou, Jiangsu, China.

Received: 7 November 2018 Accepted: 12 April 2019

Published online: 04 June 2019

\section{References}

1. Leavitt S, Ayroud M (1997) Riemerella anatipestifer infection of domestic ducklings. Can Vet J 38:113

2. Wang X, Yue J, Ding C, Wang S, Liu B, Tian M, Yu S (2016) Deletion of AS87_03730 gene changed the bacterial virulence and gene expression of Riemerella anatipestifer. Sci Rep 6:22438

3. Crasta KC, Chua KL, Subramaniam S, Frey J, Loh H, Tan HM (2002) Identification and characterization of CAMP cohemolysin as a potential virulence factor of Riemerella anatipestifer. J Bacteriol 184:1932-1939

4. Hu Q, Han X, Zhou X, Ding C, Zhu Y, Yu S (2011) OmpA is a virulence factor of Riemerella anatipestifer. Vet Microbiol 150:278-283

5. Wang X, Ding C, Wang S, Han X, Hou W, Yue J, Zou J, Yu S (2014) The AS87_04050 gene is involved in bacterial lipopolysaccharide biosynthesis and pathogenicity of Riemerella anatipestifer. PLoS One 9:e109962

6. Zou J, Wang X, Ding C, Tian M, Han X, Wang S, Yu S (2015) Characterization and cross-protection evaluation of M949_1603 gene deletion Riemerella anatipestifer mutant RA-M1. Appl Microbiol Biotechnol 99:10107-10116

7. Wang X, Liu B, Dou Y, Fan H, Wang S, Li T, Ding C, Yu S (2016) The Riemerella anatipestifer AS87_01735 gene encodes nicotinamidase PncA, an important virulence factor. Appl Environ Microbiol 82:5815-5823

8. Chang CF, Hung PE, Chang YF (1998) Molecular characterization of a plasmid isolated from Riemerella anatipestifer. Avian Pathol 27:339-345

9. Liu M, Huang M, Shui Y, Biville F, Zhu D, Wang M, Jia R, Chen S, Sun K, Zhao X, Yang Q, Wu Y, Chen X, Cheng A (2018) Roles of B739_1343 in iron 
acquisition and pathogenesis in Riemerella anatipestifer $\mathrm{CH}-1$ and evaluation of the RA-CH-1 Delta B739_1343 mutant as an attenuated vaccine. PLoS One 13:e0197310

10. Yu G, Wang X, Dou Y, Wang S, Tian M, Qi J, Li T, Ding C, Wu Y, Yu S (2016) Riemerella anatipestifer M949_1360 gene functions on the lipopolysaccharide biosynthesis and bacterial virulence. PLoS One 11:e0160708

11. McBride MJ, Nakane D (2015) Flavobacterium gliding motility and the type IX secretion system. Curr Opin Microbiol 28:72-77

12. McBride MJ, Zhu Y (2013) Gliding motility and Por secretion system genes are widespread among members of the phylum bacteroidetes. J Bacteriol 195:270-278

13. Chagnot C, Zorgani MA, Astruc T, Desvaux M (2013) Proteinaceous determinants of surface colonization in bacteria: bacterial adhesion and biofilm formation from a protein secretion perspective. Front Microbiol 4:303

14. Sato K, Yukitake H, Narita Y, Shoji M, Naito M, Nakayama K (2013) Identification of Porphyromonas gingivalis proteins secreted by the Por secretion system. FEMS Microbiol Lett 338:68-76

15. Shrivastava A, Rhodes RG, Pochiraju S, Nakane D, McBride MJ (2012) Flavobacterium johnsoniae RemA is a mobile cell surface lectin involved in gliding. J Bacteriol 194:3678-3688

16. Kharade SS, McBride MJ (2015) Flavobacterium johnsoniae PorV is required for secretion of a subset of proteins targeted to the type IX secretion system. J Bacteriol 197:147-158

17. Rhodes RG, Samarasam MN, Shrivastava A, van Baaren JM, Pochiraju S, Bollampalli S, McBride MJ (2010) Flavobacterium johnsoniae gldN and gldO are partially redundant genes required for gliding motility and surface localization of SprB. J Bacteriol 192:1201-1211

18. Rhodes RG, Samarasam MN, Van Groll EJ, McBride MJ (2011) Mutations in Flavobacterium johnsoniae sprE result in defects in gliding motility and protein secretion. J Bacteriol 193:5322-5327

19. Shrivastava A, Johnston JJ, van Baaren JM, McBride MJ (2013) Flavobacterium johnsoniae GldK, GldL, GldM, and SprA are required for secretion of the cell surface gliding motility adhesins SprB and RemA. J Bacteriol 195:3201-3212

20. Seers CA, Slakeski N, Veith PD, Nikolof T, Chen YY, Dashper SG, Reynolds EC (2006) The RgpB C-terminal domain has a role in attachment of RgpB to the outer membrane and belongs to a novel C-terminal-domain family found in Porphyromonas gingivalis. J Bacteriol 188:6376-6386

21. Guo Y, Hu D, Guo J, Wang T, Xiao Y, Wang X, Li S, Liu M, Li Z, Bi D, Zhou Z (2017) Riemerella anatipestifer type IX secretion system is required for virulence and gelatinase secretion. Front Microbiol 8:2553

22. Braun TF, Khubbar MK, Saffarini DA, McBride MJ (2005) Flavobacterium johnsoniae gliding motility genes identified by mariner mutagenesis. J Bacteriol 187:6943-6952

23. Sato K, Naito M, Yukitake H, Hirakawa H, Shoji M, McBride MJ, Rhodes RG, Nakayama K (2010) A protein secretion system linked to bacteroidete gliding motility and pathogenesis. Proc Natl Acad Sci USA 107:276-281

24. Alvarez B, Secades P, Prieto M, McBride MJ, Guijarro JA (2006) A mutation in Flavobacterium psychrophilum tlpB inhibits gliding motility and induces biofilm formation. Appl Environ Microbiol 72:4044-4053

25. Wang X, Ding C, Wang S, Han X, Yu S (2015) Whole-genome sequence analysis and genome-wide virulence gene identification of Riemerella anatipestifer strain Yb2. Appl Environ Microbiol 81:5093-5102

26. Hu Q, Zhu Y, Tu J, Yin Y, Wang X, Han X, Ding C, Zhang B, Yu S (2012) Identification of the genes involved in Riemerella anatipestifer biofilm formation by random transposon mutagenesis. PLoS One 7:e39805

27. Hunnicutt DW, McBride MJ (2000) Cloning and characterization of the Flavobacterium johnsoniae gliding-motility genes gldB and gldC. J Bacteriol 182:911-918
28. Hunnicutt DW, McBride MJ (2001) Cloning and characterization of the Flavobacterium johnsoniae gliding motility genes gldD and gldE. J Bacteriol 183:4167-4175

29. Newton J, Wood T, Marlenehartley M (1997) Isolation and partial characterization of extracellular proteases produced by isolates of Flavobac terium columnare derived from channel catfish. J Aquat Anim Health 9:75-85

30. Kim D, Langmead B, Salzberg SL (2015) HISAT: a fast spliced aligner with low memory requirements. Nat Methods 12:357-360

31. Wilson GW, Stein LD (2015) RNASequel: accurate and repeat tolerant realignment of RNA-seq reads. Nucleic Acids Res 43:e122

32. Kim D, Pertea G, Trapnell C, Pimentel H, Kelley R, Salzberg SL (2013) TopHat2: accurate alignment of transcriptomes in the presence of insertions, deletions and gene fusions. Genome Biol 14:R36

33. Trapnell C, Williams BA, Pertea G, Mortazavi A, Kwan G, van Baren MJ, Salzberg SL, Wold BJ, Pachter L (2010) Transcript assembly and quantification by RNA-Seq reveals unannotated transcripts and isoform switching during cell differentiation. Nat Biotechnol 28:511-515

34. Huggett J, Dheda K, Bustin S, Zumla A (2005) Real-time RT-PCR normalisation; strategies and considerations. Genes Immun 6:279-284

35. Dou Y, Wang X, Yu G, Wang S, Tian M, Qi J, Li T, Ding C, Yu S (2017) Disruption of the M949_RS01915 gene changed the bacterial lipopolysaccharide pattern, pathogenicity and gene expression of Riemerella anatipestifer. Vet Res 48:6

36. Nelson SS, Glocka PP, Agarwal S, Grimm DP, Mcbride MJ (2007) Flavobacterium johnsoniae SprA is a cell surface protein involved in gliding motility. J Bacteriol 189:7145

37. Saiki K, Konishi K (2007) Identification of a Porphyromonas gingivalis novel protein sov required for the secretion of gingipains. Microbiol Immunol 51:483-491

38. Kharade SS, McBride MJ (2014) Flavobacterium johnsoniae chitinase ChiA is required for chitin utilization and is secreted by the type IX secretion system. J Bacteriol 196:961-970

39. Lasica AM, Ksiazek M, Madej M, Potempa J (2017) The type IX secretion system (T9SS): highlights and recent insights into its structure and function. Front Cell Infect Microbiol 7:215

40. Tomek MB, Neumann L, Nimeth I, Koerdt A, Andesner P, Messner P, Mach L, Potempa JS, Schaffer C (2014) The S-layer proteins of Tannerella forsythia are secreted via a type IX secretion system that is decoupled from protein O-glycosylation. Mol Oral Microbiol 29:307-320

41. Li N, Zhu Y, LaFrentz BR, Evenhuis JP, Hunnicutt DW, Conrad RA, Barbier P, Gullstrand CW, Roets JE, Powers JL, Kulkarni SS, Erbes DH, Garcia JC, Nie P, McBride MJ (2017) The type IX secretion system is required for virulence of the fish pathogen Flavobacterium columnare. Appl Environ Microbiol 83:e01769-17

42. Shi Y, Ratnayake DB, Okamoto K, Abe N, Yamamoto K, Nakayama K (1999) Genetic analyses of proteolysis, hemoglobin binding, and hemagglutination of Porphyromonas gingivalis. Construction of mutants with a combination of rgpA, rgpB, kgp, and hagA. J Biol Chem 274:17955-17960

43. Taguchi Y, Sato K, Yukitake H, Inoue T, Nakayama M, Naito M, Kondo Y, Kano K, Hoshino T, Nakayama K, Takashiba S, Ohara N (2016) Involvement of an Skp-Like protein, PGN_0300, in the Type IX secretion system of Porphyromonas gingivalis. Infect Immun 84:230-240

\section{Publisher's Note}

Springer Nature remains neutral with regard to jurisdictional claims in published maps and institutional affiliations. 